\title{
State-of-the-art energetic and morphological modelling of the launching site of the M87 jet
}

Alejandro Cruz-Osorio ${ }^{1 *}$, Christian M. Fromm ${ }^{2,1,3 *}$, Yosuke Mizuno ${ }^{4,1 *}$, Antonios Nathanail ${ }^{5,1}$, Ziri Younsi ${ }^{6}$, Oliver Porth ${ }^{7}$, Jordy Davelaar ${ }^{8,9,10}$, Heino Falcke ${ }^{10,3}$, Michael Kramer ${ }^{3,11}$ and

Luciano Rezzolla $a^{1,12,13}$

${ }^{1}$ Institut für Theoretische Physik, Goethe Universität, Frankfurt, Germany

${ }^{2}$ Black Hole Initiative at Harvard University, Cambridge, MA, USA

${ }^{3}$ Max-Planck-Institut für Radioastronomie, Bonn, Germany

${ }^{4}$ Tsung-Dao Lee Institute and School of Physics and Astronomy, Shanghai Jiao Tong University, Shanghai, People's Republic of China

${ }^{5}$ Department of Physics, National and Kapodistrian University of Athens, Athens, Greece

${ }^{6}$ Mullard Space Science Laboratory, University College London, Dorking, UK

${ }^{7}$ Anton Pannekoek Institute for Astronomy, University of Amsterdam, Amsterdam, The Netherlands

${ }^{8}$ Department of Astronomy and Columbia Astrophysics Laboratory, Columbia University, New York, NY, USA

${ }^{9}$ Center for Computational Astrophysics, Flatiron Institute, New York, NY, USA

${ }^{10}$ Department of Astrophysics/IMAPP, Radboud University Nijmegen, Nijmegen, The Netherlands

${ }^{11}$ Jodrell Bank Centre for Astrophysics, University of Manchester, Manchester, UK

${ }^{12}$ Frankfurt Institute for Advanced Studies, Frankfurt, Germany

${ }^{13}$ School of Mathematics, Trinity College, Dublin, Ireland

M 87 has been the target of numerous astronomical observations across the electromagnetic spectrum and Very Long Baseline Interferometry (VLBI) resolved an edge-brightened jet ${ }^{1-4}$. However, the origin and formation of its jets remain unclear. In our current understand black holes (BH) are the driving engine of jet formation ${ }^{5}$, and indeed the recent Event Horizon Telescope (EHT) observations revealed a ring-like structure in agreement with theoretical models of accretion onto a rotating Kerr $\mathrm{BH}^{6}$. In addition to the spin of the $\mathrm{BH}$ being a potential source of energy for the launching mechanism, magnetic fields are believed to play a key role in the formation of relativistic jets ${ }^{7,8}$. A priori, the spin, $a_{\star}$, of $\mathrm{BH}$ in ${\mathrm{M} 87^{\star}}^{\text {is }}$ unknown, however, when accounting for the estimates on the X-ray luminosity and jet power, values $\left|a_{\star}\right| \gtrsim 0.5$ appear favoured ${ }^{6}$. Besides the properties of the accretion flow and the BH spin, the radiation microphysics including the particle distribution (thermal ${ }^{6}$ and nonthermal $^{9,10}$ ) as well as the particle acceleration mechanism ${ }^{11}$ play a crucial role. We show that general-relativistic magnetohydrodynamics simulations and general-relativistic radiative transfer calculations can reproduce the broadband spectrum from the radio to the nearinfrared regime and simultaneously match the observed collimation profile of $M 87$, thus allowing us to set rough constraints on the dimensionless spin of M87* to be $0.5 \lesssim a_{\star} \lesssim 1.0$, with higher spins being possibly favoured. 
We here report the results of long-term, high-resolution, three-dimensional (3D) generalrelativistic magnetohydrodynamic (GRMHD) simulations of magnetically arrested disks (MADs) disks around rotating BHs with different spin values, that is, $a_{\star}=\{-0.9375,-0.5,0.0,0.5,0.9375\}$. A particularly important aspect of our work is the inclusion of a non-thermal energy contribution, which we model in terms of a "kappa" distribution function for the electron population" ${ }^{12,13}$, together with a sub-grid model that aims at modelling the effects introduced by magnetic reconnection ${ }^{14}$. In this way, we combine 3D MAD GRMHD simulations with a hybrid thermal-nont-hermal particle distribution function to model the observed innermost structure of the relativistic jet in M87.

The first step in our modelling involves the simulation of the plasma dynamics around the rotating BHs. To this scope, we initialise our state-of-the-art three-dimensional GRMHD simulations with a magnetised torus seeded with weak poloidal magnetic field in hydrodynamical equilibrium using the numerical code BHAC. The development of the magnetorotational (MRI) instability triggers accretion and leads to a fully turbulent flow after a timescale $t=10,000 M \simeq 10 \mathrm{yr}$, where $M=6.5 \times 10^{9} M_{\odot}$ is the mass of M87*. At this point, a stationary MAD solution is attained, with a the dimensionless magnetic flux onto the $\mathrm{BH}$ reaches values $\Psi:=\Phi_{\mathrm{BH}} / \sqrt{\dot{M}} \sim 15$, where $\dot{M}$ is the mass accretion rate and $\Phi_{\mathrm{BH}}$ the magnetic flux across the horizon. Three main regions characterise the flow: a bound, dense and weakly magnetised accretion disk; an unbound and slow disk wind; and a diluted, highly magnetised funnel region near the polar axis, typically referred as the "jet" (see Fig. 1 for a 3D rendering of the simulation, and Extended Data Figures 1 and 2).

The second step requires the calculation of the radiative signatures and, to this scope, the general-relativistic radiative transfer (GRRT) calculations performed with the BHOSS code ${ }^{15}$ need to be tuned by fixing the mass of the $\mathrm{BH}$ to that of $\mathrm{M} 87^{*}$ and the distance to $D=16.8 \mathrm{Mpc}$. In addition, the energy distribution function of the emitting electrons needs to be specified and the mass accretion rate adjusted to match the observed flux densities. As anticipated, we employ a kappa distribution function, which consists of a thermal low-energy core and of a non-thermal high-energy tail to include the contribution of the magnetic energy to the heating of the particles. Also, since the GRMHD simulations model the dynamics of the non-radiating ions, a prescription is needed to relate the temperature of the latter to the temperature of the radiating electrons. For this, we use the so-called "R- $\beta$ " parameterisation ${ }^{16}$, in which the temperatures of the two fluids are related in terms of a free parameter " $\mathrm{R}$ " and of the ratio between the gas and magnetic pressures, i.e., the plasma $\beta$ function.

To ensure a stable mass accretion rate and a well-developed turbulence, we only consider the results of the simulations in the time interval between 13,000 - 15,000 $M$ for our GRRT analysis. We scale the mass accretion rate and adjust the additional parameters of the electron kappa-distribution function in order to match the observed compact flux density of $\sim 1.0 \mathrm{Jy}$ at $230 \mathrm{GHz}^{17}$ and to fit the broad-band spectrum of the flux density $S$ in the range of frequencies $\left.\nu, 10^{10} \mathrm{~Hz} \leq \nu \leq 10^{16} \mathrm{~Hz}\right)$. The additional parameters that need to be taken into account are: the fraction of magnetic energy contributing to the heating of the radiating electrons, $\epsilon$, and the injection radius of the accelerated particles, $r_{\mathrm{inj}}=10 \mathrm{M}$. In Fig. 2 we present the results of the 


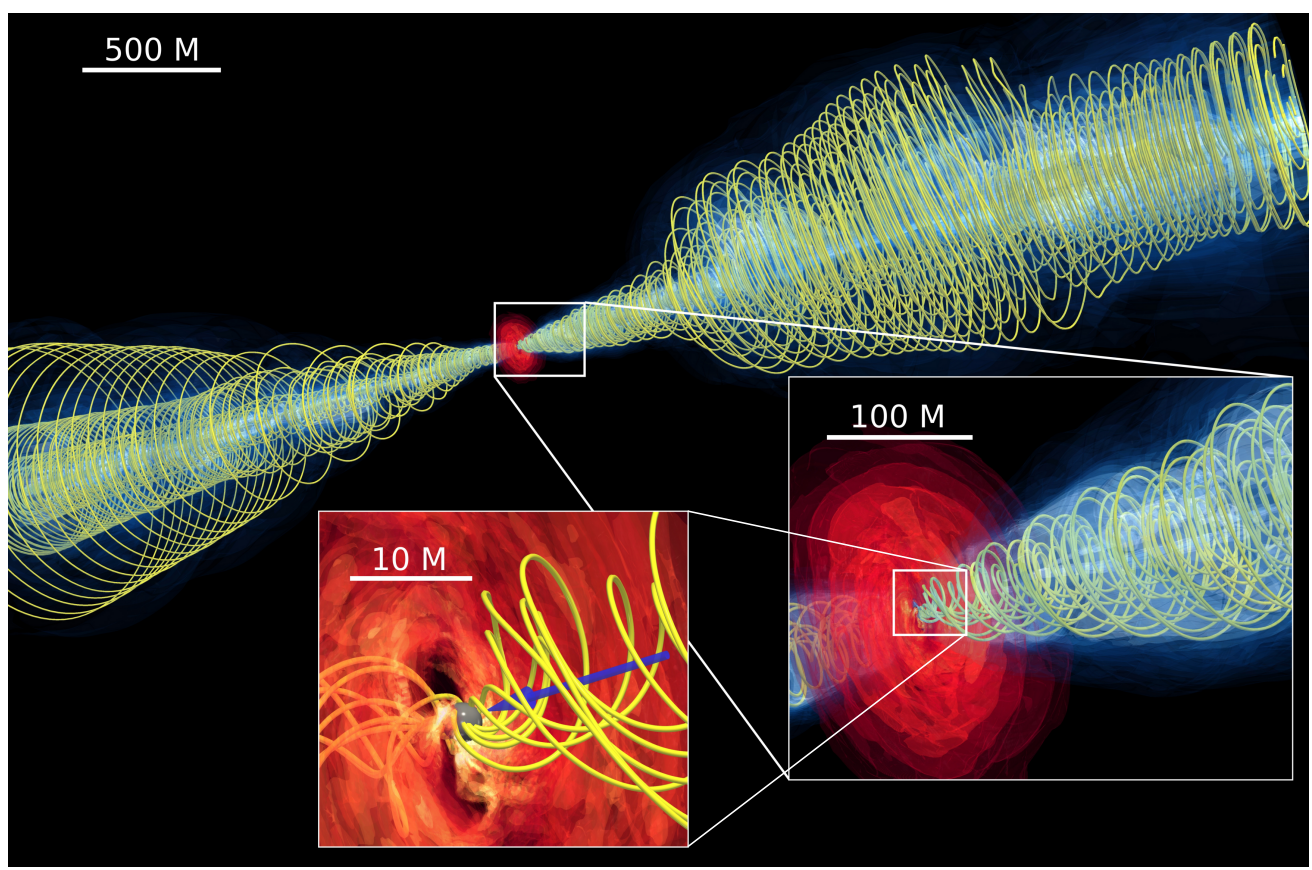

Figure 1: Large-scale 3D morphology of the jet and disk from a 3D GRMHD MAD simulation for a BH with $a_{\star}=\mathbf{0 . 9 3 7 5}$. The image shows the fast, dilute and highly magnetised out-flowing jet (blue) and the denser and weakly magnetised accretion disk (red). Magnetic field lines spiral around the jet are plotted in yellow. The insets offer a magnification of the large-scale image on the reported scales; the $\mathrm{BH}$ and disk angular momenta point in the bottom-left direction (blue arrow).

spectrum obtained from the simulation that provides the best-fit to the observed spectrum for $\mathrm{BH}$ spins $a_{\star}=0.5$ and $a_{\star}=0.9375$ (for all range of BH spins see Extended Data Figure 3). Different symbols indicate the observational data grouped into ten-year periods ranging from 1990 to 2020 (refs. ${ }^{3,9,17,18,18-25}$ ).

The best-fit was obtained when using $\epsilon=0.5$ and $r_{\text {inj }}=10 \mathrm{M}$, where the latter was chosen to match the location of the stagnation surface, which is typically found between $5 M$ and $10 M$ (ref. ${ }^{26}$ ). In addition, to avoid the numerical contamination of our results from the highly magnetised regions of the funnel where the GRMHD equations do not provide an accurate description of the plasma, we exclude regions with a very large magnetisation, setting a cut-off at $\sigma_{\text {cut }}=3$.

Clearly, Fig. 2 shows that the combination of MAD GRMHD simulations together with an electron kappa-distribution function and a magnetic reconnection sub-grid model, enables us to obtain a remarkably good fit of the whole spectrum of M87, both in the radio $\left(10^{10} \mathrm{~Hz} \leq \nu \leq\right.$ $\left.10^{12} \mathrm{~Hz}\right)$ and in the near-infrared $\left(10^{14} \mathrm{~Hz} \leq \nu \leq 10^{15} \mathrm{~Hz}\right)$ bands. The novel result is that we have good agreement with the spectrum, accompanied by a good match to the jet morphology in its launching site. 


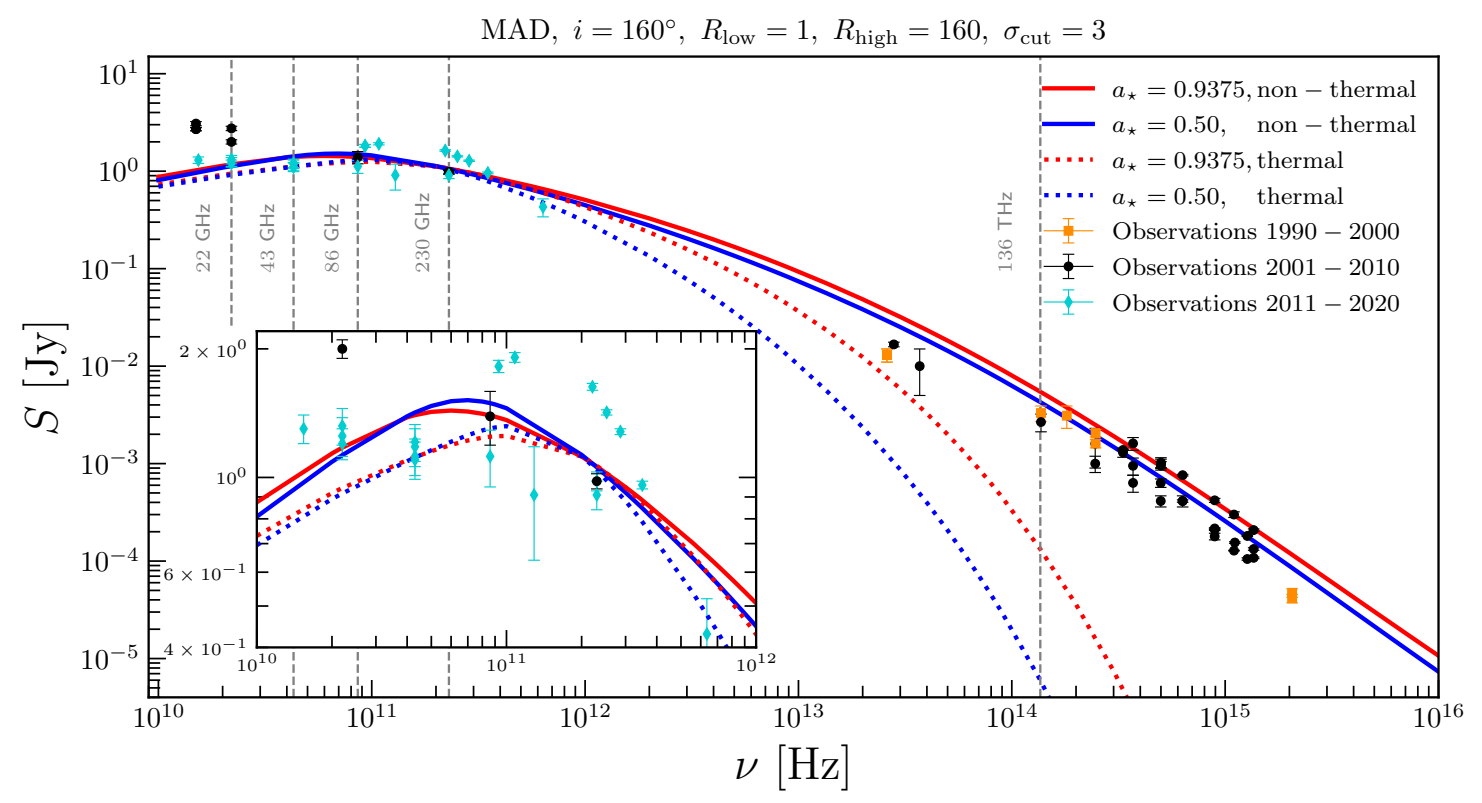

Figure 2: Broadband spectrum of the flux density of M87. Solid lines show the average spectra from simulations (blue for $a_{\star}=0.50$ and red for $a_{\star}=0.9375$ ) as computed between 13,000 M and $15,000 M$ for non-thermal models with $\epsilon=0.5$ at an inclination angle of $i=160^{\circ}$, whereas dotted lines correspond to thermal models. Gray vertical lines show the most representative frequencies. The observational data is indicated with symbols and covers three decades grouped into ten-years windows: orange squares for 1990-2000, black circles for 2001-2010, and turquoise-diamonds for 2011-2020 (refs. ${ }^{3,9,17,18,18-25}$ ). For each observational data, the uncertainties indicate the variability during the observations.

In addition to the broad-band spectrum, our simulations can reproduce the morphology of the M87 jet with high accuracy up to distances $r \sim 1$ mas from the core, as deduced from the Global mm Very Long Baseline Interferometry Array (GMVA) observation in February $2014{ }^{9}$. To perform such a comparison, we generate 200 GRRT images covering a time span of 2,000 $\mathrm{M}$ at $86 \mathrm{GHz}(3 \mathrm{~mm})$. Furthermore, we limit the dynamical range of the flux of our synthetic images to be within three orders of magnitude from the maximum flux, i.e., $\log _{10} S \in[-7.1,-4.1] \mathrm{Jy} /$ pixel in agreement with the GMVA observations. In Fig. 3 we present the best-fit images - as deduced from the normalised cross-correlation coefficient and structural-dissimilarity index ${ }^{27}$ computed between the GRRT images and the GMVA observations. The top two images show the high-resolution simulations, whereas the associated bottom two images show the result of the convolve of the simulations with a beam of $116 \times 307 \mu$ as to mimic the limited resolution of the GMVA observations. The 2014 GMVA image is shown on the right. Note that both models produce large opening angles, but also that the characteristic "limb brightening" of the 2014 GMVA image is visible and closely similar only for the $a_{\star}=0.9375$ model. Furthermore, the rapidly spinning BH also displays a detectable signature of a counter jet, again in good in agreement with the 2014 GMVA observations (Extended Data Figures 4 and 5 also show thermal model and averaged images). 


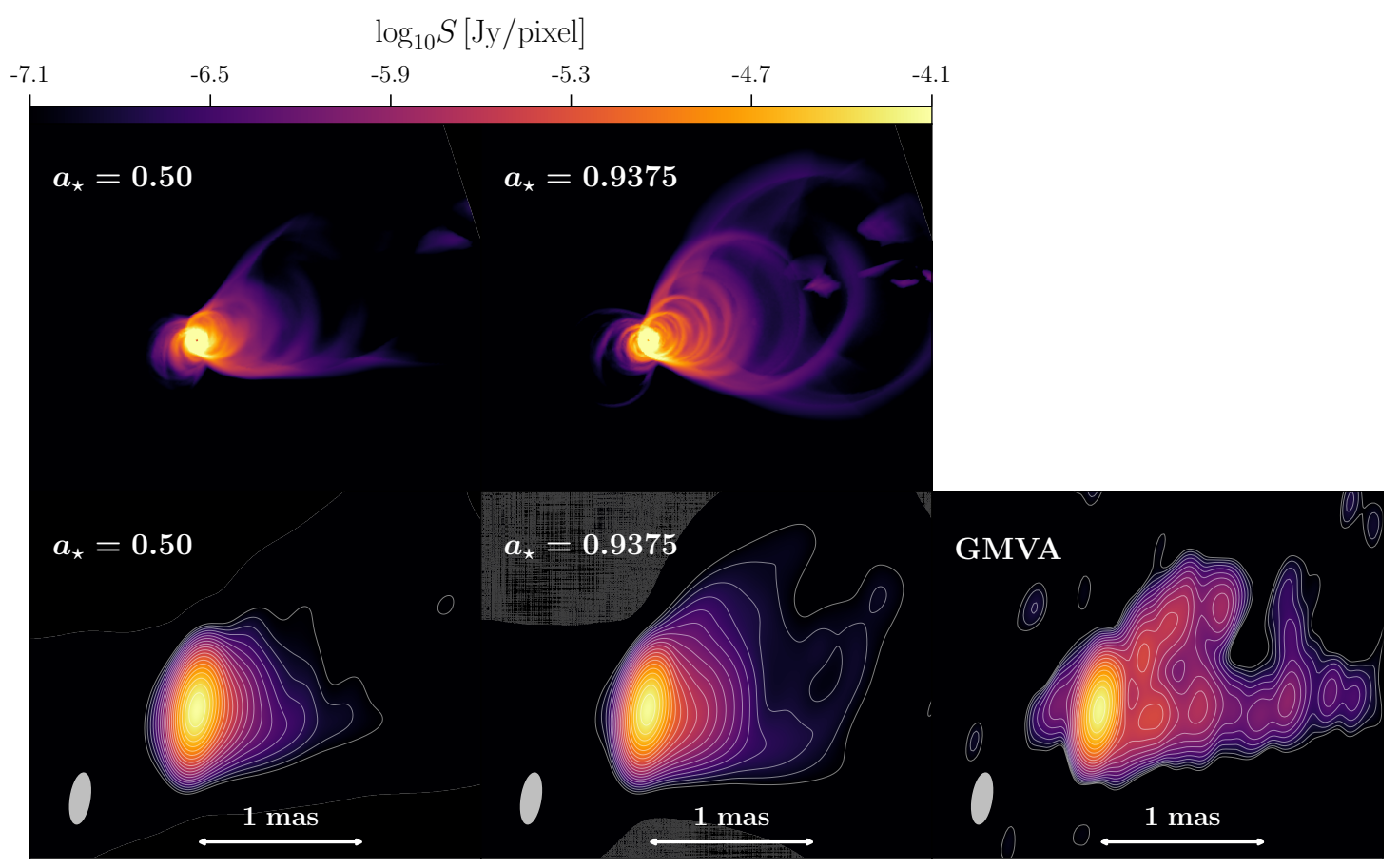

Figure 3: Morphological comparison between observations and theoretical models. The top two images report the GRRT results for $a_{\star}=0.50$ (left) and $a_{\star}=0.9375$ (middle) at $86 \mathrm{GHz}$. The associated bottom two images show the corresponding convolved images, and the image on the right offers the GMVA observations. The convolving beam of $116 \times 307 \mu$ as at a position angle of $-9^{\circ}$ is shown in the lower-left corner. We limit the dynamical ranges of the images to three orders of magnitude, in agreement with current GMVA observations.

A more quantitative measure of the properties of the jet structure as obtained from the observations and from simulations can be obtained by measuring the jet diameter. For this scope, we slice the jet in a direction orthogonal to that of propagation and fit the flux-density profile using up to three different Gaussians. The jet width is then computed as the distance between two outermost Gaussians and this shown in Fig. 4 for various spins. Note that at small distances, i.e., for $r<0.2$ mas, the two models with different spins yield jet widths that are smaller than the one deduced from GMVA observations of M87. However, for larger distances, i.e., for 0.2 mas $\leq r \leq 0.7$ mas, the jet widths are in good agreement with the observations for both spins; this morphological match is lost for simulations with $a_{\star} \lesssim 0.5$ ( see Extended Data Figure 6 for a quantitative measurement).

In summary, the results presented here clearly show that long-term, high-resolution and stateof-the-art GRMHD simulations of magnetically arrested disks in combination with a non-thermal description of the electron energy distribution can reproduce not only the broad-band spectrum, but also the $86 \mathrm{GHz}$ emission structure of M87. Interestingly, if non-thermal particles are included in 


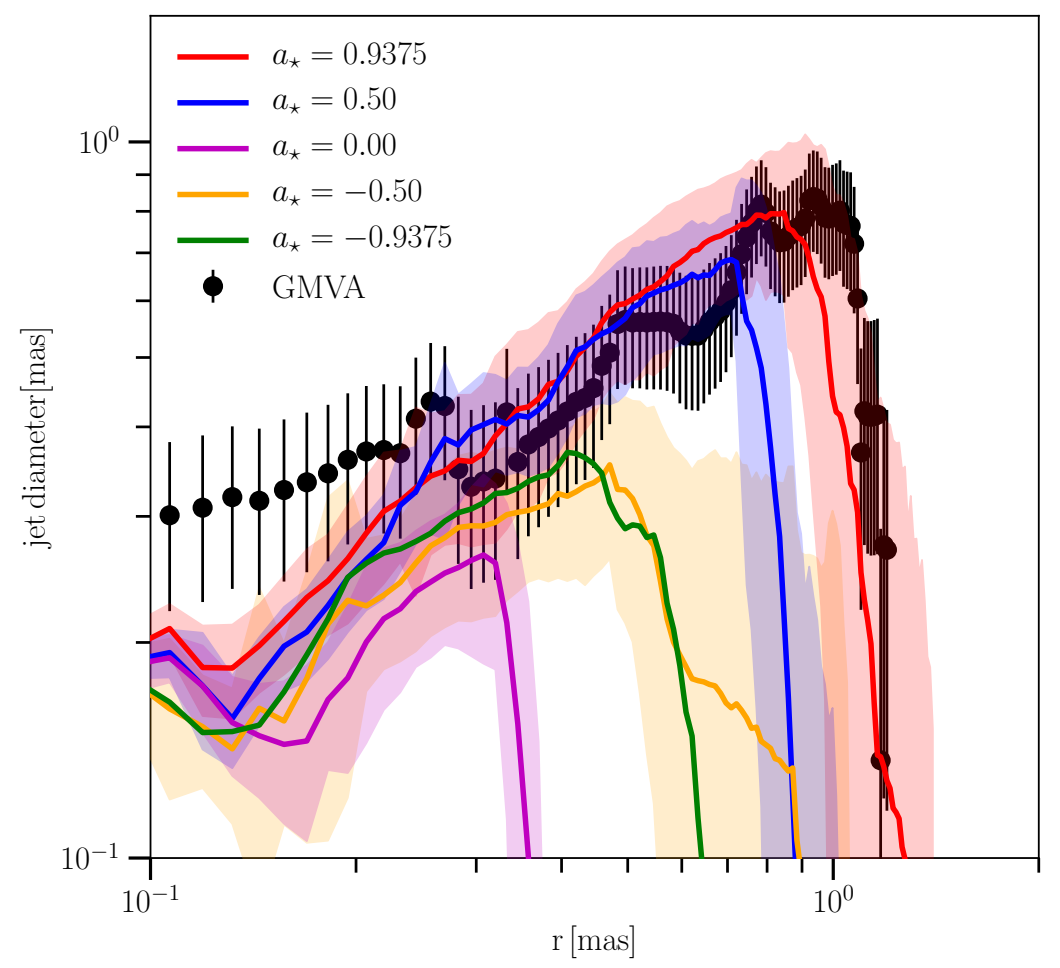

Figure 4: Jet-diameter comparison between observations and theoretical models. Shown with black points is the jet width computed from the GMVA observation where the uncertainties are obtained by assuming an uncertainty of $1 / 4$ of the beam size at the $r=0$ and a linear increase until $1 / 2$ of the beam size is reached at $r=2$ mas (ref. ${ }^{42}$ ). Reported instead with solid lines are the equivalent measures from the convolved GRRT images covering a time span of 2,000 $M$ (green for $a_{\star}=-0.9375$, orange for $a_{\star}=-0.50$, magenta for $a_{\star}=0.00$, red for $a_{\star}=0.50$ and blue for $a_{\star}=0.9375$ ); shown as shaded regions are variations within the standard deviation.

the GRRT calculations, both models are able to reproduce the observed spectrum of M87, including a flat spectrum in the radio $\left(10^{10} \mathrm{~Hz} \leq \nu \leq 10^{12} \mathrm{~Hz}\right)$ and in the near-infrared $\left(10^{14} \mathrm{~Hz} \leq \nu \leq\right.$ $10^{15} \mathrm{~Hz}$ ) bands. Furthermore, since both MAD models naturally produce jets with large opening angles, the simulations provide a remarkably good match of the morphology of the jet as measured in terms of the diameter along the direction of propagation. Because this match is lost for BHs with small or negative spins, our results constrain the dimensionless spin of M87* to be roughly in the range $0.5 \lesssim a_{\star} \lesssim 1.0$, with higher spins being possibly favoured.

The promising results presented here call for additional work and improvements, where modelling including particle heating, radiative cooling and polarisation will further improve the ability of our models to reproduce also finer details of the observed features of the M87 jet. In addition, the ongoing technical improvements of the Very Long Baseline Interferometry technique will allow us to image the jet footpoint with unprecedented resolution and further constrain our models. 


\section{Methods}

\section{General-relativistic magnetohydrodynamic simulations of magnetically arrested disks.}

The simulations presented in the main text were performed using the state-of-the-art multidimensional GRMHD code BHAC V1. $1^{28}$, which has been designed to solve the equations of GRMHD in arbitrary but fixed spacetimes and different coordinate systems; the results presented here refer to 3D simulations using spherical polar coordinates. The numerical techniques are based on second-order finite-volume and high-resolution shock-capturing methods ${ }^{29}$, complemented with flux-constrained transport of the magnetic field so as to preserve its divergence-free constraint $\nabla \cdot \mathbf{B}=0$ (ref. ${ }^{30}$ ). We have carried out simulations considering that the spacetime is described by the Kerr solution written in Kerr-Schild coordinates with dimensionless spin parameter $a_{\star}=\{-0.9375,-0.5,0.0,0.5,0.9375\}$. Most of our attention, however, is concentrated on the cases of positively and rapidly spinning BHs, i.e., $a_{\star}=0.5$ and $a_{\star}=0.9375$, as these are the configurations that provide the best morphological match to the GMVA observations.

As initial data for our 3D GRMHD simulations, we consider a magnetised torus with constant specific angular momentum, $\ell:=u_{\phi} / u_{t}=6.8\left(a_{\star}=0.5\right)$ and $6.76\left(a_{\star}=0.9375\right)$ in hydrodynamic equilibrium around the rotating $\mathrm{BH}$, where $\boldsymbol{u}$ is the fluid four-velocity. A weak, single-loop poloidal magnetic field is added on the hydrodynamical solution in terms of the vector potential $A_{\phi} \propto \max (q-0.2,0)$, where $q:=\left(\rho / \rho_{\max }\right)\left(r / r_{\text {in }}\right)^{3} \sin ^{3} \theta \exp (-r / 400)$, and normalised so that $\beta_{\min }=\left(2 p / b^{2}\right)_{\min }=100$, where the subscript min refers to the minimum value inside the torus. The location of the inner edge and central radius of the torus, defined by the equipotential surfaces of the gravitational-centrifugal potential, are $r_{\text {in }}=20 M$ and $r_{\text {centre }}=40 \mathrm{M}$, respectively ${ }^{29,31}$. We make use of an ideal-gas equation of state ${ }^{29}$ with an adiabatic index of $\Gamma=4 / 3$ to model the relativistic gas in the torus. As is customary in this type of simulations, we excite the magnetorotational instability in the torus by adding a $4 \%$ random perturbation to the equilibrium gas pressure of the torus. To avoid numerical failures due to the presence of vacuum regions outside the torus, it is conventional to add an "atmosphere" to regions which should be virtually in vacuum. For all numerical cells whose rest-mass density satisfies the condition $\rho \leq \rho_{\mathrm{fl}}$, or whose gas pressure is such that $p \leq p_{\mathrm{fl}}$, we simply set $\rho=\rho_{\mathrm{fl}}$ and $p=p_{\mathrm{fl}}$, where the floor values are $\rho_{\mathrm{fl}}:=10^{-4} r^{-3 / 2}$ and $p_{\mathrm{fl}}:=\left(10^{-6} / 3\right) r^{-5 / 2}$ and are based on the exact solution of the Bondi-accretion problem.

The spherical polar (spatial) coordinate map $(r, \theta, \phi)$ covers the simulation domain in the range $r \in\left[0.8 r_{\mathrm{EH}}, 2500 M\right], \theta \in[0, \pi]$, and $\phi \in[0,2 \pi]$, where $r_{\mathrm{EH}}=M+\sqrt{M-a_{\star}^{2}}$ is the event-horizon radius, and with the innermost cell well inside the event horizon. The grid spacing is logarithmic in the radial direction and uniform in the two angular $\theta-$ and $\phi$ - directions, using three refinement levels with the effective number of grid points given by $\left(N_{r}, N_{\theta}, N_{\phi}\right)=(384,192,192)$. At the inner radial boundary, which is within the event horizon thanks to the horizon-penetrating coordinates, we apply an inflow boundary condition, while at the outer radial boundary we enforce an outflow boundary condition by performing a copy of the physical variables. At the polar boundaries, i.e., for $\theta=0$ and $\theta=\pi$ we apply the so-called "hard" polar boundary conditions, namely, we assume that a solid reflective wall is present along the polar boundaries, where the flux 
and poloidal velocity through the boundaries is set to be zero, adjusting the electromotive force in the constrained transport routine ${ }^{30,32}$. For the azimuthal direction, instead, periodic boundary conditions are employed in all physical variables across the cells at $\phi=0$.

\section{General-relativistic radiation-transfer with a non-thermal emission model.}

In order to compute the radio images of the accretion flow around M87* and of the corresponding jet, we have employed the GRRT code BHOSS ${ }^{15,33}$. In this code, electromagnetic radiation propagates along null geodesics, which are used to solve the radiative-transfer equation along such path $^{34}$. To generate large number of images over a considerable time range, we consider for all simulations the time interval $t \in[13,000 M-15,000 M]$, with a cadence of $10 \mathrm{M}$; the resulting 200 images cover about two years of observations given the estimated mass of M87*, which we take to be $6.5 \times 10^{9} \mathrm{M}_{\odot}$ (ref. ${ }^{35}$ ). The image size is then also computed adopting a distance from M87* of $16.8 \mathrm{Mpc}$ (ref. ${ }^{35}$ ).

The electron temperature in the jet is computed through the temperature of the ions in the so-called "two-temperatures" model so that the ion-to-electron temperature ratio is expressed as $T_{\mathrm{i}} / T_{\mathrm{e}}:=\left(R_{\text {low }}+R_{\text {high }} \beta^{2}\right) /\left(1+\beta^{2}\right)$ ( ref. $\left.{ }^{36}\right)$, which depends on the ratio between the gas and magnetic pressures, i.e., the plasma $\beta$ function, which is large inside the accretion flow and small elsewhere. The relation between the two temperatures also makes use of two free coefficients, $R_{\text {low }}$ and $R_{\text {high }}$, whose values for regions involving the emission from the jet $(\beta \ll 1)$, and from the disk $(\beta \gg 1)$, have been set to be $R_{\text {low }}=1$ and $R_{\text {high }}=160$, respectively. These values are routinely adopted in the two-temperatures model and we have systematically verified that they also provide the best match to the broadband spectrum and to the jet morphology.

An essential aspect of our GRRT modelling consists in the use of a non-thermal energy distribution for the electrons that we model, following Davelaar et al. (2019) ${ }^{13}$, in terms of the so-called "kappa" distribution, which is a combination of a thermal electron population and of a population with a power-law energy distribution, i.e., $d n_{\mathrm{e}} / d \gamma_{\mathrm{e}}=N \gamma_{\mathrm{e}} \sqrt{\gamma_{\mathrm{e}}^{2}-1}\left[1+\left(\gamma_{\mathrm{e}}-1\right) /(\kappa w)\right]^{-(\kappa+1)}$, where $\gamma_{\mathrm{e}}$ is the Lorentz factor of the electrons and $N$ is a normalization parameter. From a more phenomenological point of view, the so-called kappa-jet model intends to describe the energy contribution of electrons accelerated due to magnetic reconnection at the base of the jet. The weighted temperature $w$ is defined as $w:=(\kappa-3) \Theta_{\mathrm{e}} / \kappa+\frac{1}{2} \epsilon\left[1+\tanh \left(r-r_{\text {inj }}\right)\right](\kappa-3) m_{\mathrm{p}} \sigma /\left(6 \kappa m_{\mathrm{e}}\right)$, where $r_{\mathrm{inj}}=10 \mathrm{M}$ is the injection position, $\Theta_{e}$ is the dimensionless electron temperature, $m_{\mathrm{p}}$ and $m_{\mathrm{e}}$ are respectively the proton and electron masses, $\sigma$ is the magnetisation parameter, i.e., the ratio of the magnetic and rest-mass energy densities, and $\epsilon$ is a tunable parameter between zero and one that accounts for the fraction of magnetic energy contributing to the heating of the radiating electrons. Finally, the power-law index $\kappa$, depends on the microphysics of the plasma and the expression employed here, namely $\kappa:=2.8+0.7 \sigma^{-1 / 2}+3.7 \sigma^{-19 / 100} \tanh \left(23.4 \sigma^{26 / 100} \beta\right)$, is inspired by particle-in-cell simulations ${ }^{11,13}$ and is set to be a function of magnetisation parameters $\beta$ and $\sigma$ only.

Extended Data Figures 1 and 2 report the large-scale morphology of the jet from GRMHD 
simulations of a Kerr BH with spin $\mathbf{a}_{\star}=\mathbf{0 . 9 3 7 5}$. Extended Data Figure 1 concentrates on the large-scale structure of the jet, whereas Extended Data Figure 2 offers a magnification near the event horizon. In both cases, shown from left to right are the magnetisation parameters $\sigma$, the electron temperature $T_{\mathrm{e}}$, the distribution of the power-law index $\kappa$, and the weighted temperature $w$. All quantities are averaged in space (over the azimuthal direction) and in time (over a time interval of 2,000 $M$ with a cadence of $10 M$ ). The white dashed line marks the boundary between bounded (i.e., matter with Bernoulli parameter Be $:=-h u_{t}<1.02$, where $h$ is the specific enthalpy ${ }^{29}$ ) and unbounded $(\mathrm{Be}>1.02)$ material, whereas the black lines show the most important contours of the logarithm of the magnetisation. Also reported away from the polar axis are the contours corresponding to $\sigma=3.0$, which we take to separate the "jet spine" (defined as $\sigma>\sigma_{\max }:=3.0$ ), from the "jet sheath", (for which $0.1<\sigma<3.0$ ), and from the "external wall" (defined as $\sigma=0.1$ ).

To compute the mass accretion rate, we normalise the emission at a resolution of $800 \times 800$ pixels - which corresponds to a field of view of 4 mas $\sim 10^{3} M$ - so as to reproduce the observed flux of M87 at $230 \mathrm{GHz}$ of $\simeq 1.0 \mathrm{Jy}$ (refs. ${ }^{17,21}$ ), excluding the emission from the jet-spine region, i.e., regions with $\sigma>3.0$. The resulting mass accretion rates are $\dot{M}=3.33 \times 10^{-4} M_{\odot} \mathrm{yr}^{-1}$ and $\dot{M}=1.06 \times 10^{-4} M_{\odot} \mathrm{yr}^{-1}$ for the two rapidly rotating BHs with $a_{\star}=0.5$ and $a_{\star}=0.9375$, respectively. The inclination angle of the observation is assumed to be fixed at $160^{\circ}$, as deduced from the recent observations of the $\mathrm{EHT}^{6}$.

To constrain our numerical simulations and extract information on the free parameters of our models, we have used historical observations of the broadband spectrum of M87 as well as the jet morphology in terms of the jet opening angle and jet width measured from the core and along the direction of propagation. After having fixed the BH spin and the jet-spine wall, i.e., after having set a value for $\sigma_{\text {cut }}$, we have explored the electron-temperature parameters by varying the values of the free coefficients $R_{\text {low }}$ and $R_{\text {high }}$ as a way to heat or cool the electrons in the jet funnel. In this way, we have found that large-scale, coherent jet morphology can be imaged only for large values of $R_{\text {high }}$, e.g., $R_{\text {high }}=160$, quite independently of the electron distribution function, i.e., whether a non-thermal emission is included or not.

However, although not important for the morphology, the inclusion of a non-thermal component is important to obtain a good match with the observed spectrum and in the near-infrared, in particular. Hence, after finding the optimal description for the electron temperature with $R_{\text {low }}=1$ and $R_{\text {high }}=160$, we have explored the magnetic-energy contribution in the non-thermal component by varying the parameter $\epsilon$ for fixed values of the BH spin and of $\sigma_{\text {cut }}$. In this way, we found good a agreement with jet morphology for $0.25 \lesssim \epsilon \lesssim 1$, noting, however that the near-infrared flux is over-produced with respect to the observations $\epsilon \geq 0.75$. At the same time, we have explored different locations of the jet-spine wall by changing the cut-off value for the magnetisation $\sigma_{\text {cut }}$, see also Chael et al. (2019) ${ }^{37}$. In this way, it was possible to determine that the highly magnetised region in the jet spine contributes substantially to the near-infrared emission, so that a combined good match of the observed broadband spectrum and of the morphology can be obtained for $3 \lesssim \sigma_{\text {cut }} \lesssim 5$. We also performed the GRRT calculations for different BH spins fixing the electron temperature parameters, the location of the jet-spine wall, and the parameters of the 
non-thermal energy contribution, as discussed above. In this way we found only small variations in the radio and near-infrared bands when changing the spin of the $\mathrm{BH}$ from co-rotating to counterrotating cases. The average thermal and non-thermal spectra for the best-fit parameters have been presented in Extended Data Figure 3 for the whole range of BH spins considered here. Moreover, because small and counter-rotating BHs show small opening angles and very short jets, a good agreement with the observed jet morphology can be achieved only for $0.5 \lesssim a_{\star} \lesssim 1.0$. This is shown in Extended Data Figure 4 that reports the GRRT images (top row) and the convolved ones (bottom row) for thermal and non-thermal emission models, and for two BHs with spins $a_{\star}=0.50$ and $a_{\star}=0.9375$, respectively. The images refer to a representative time $t=13,820 \mathrm{M}$, and we show as an ellipse in the lower-left corner the convolving beam with axes $116 \times 307 \mu$ as, as in the observational data. To remark that these features are not dependent on the specific choice of the image frame, we show in Extended Data Figure 5 the same same quantities as in Extended Data Figure 4 but after performing and average in time between 13,000 $M$ and 15,000 M. Clearly, all of the features discussed in Extended Data Figure 4 for a single snapshot are present also when averaging in time; a more detailed discussion of this point will be presented elsewhere.

In summary, when combining all the constraints deduced for the electron temperature, the jet spine, the magnetic energy in the non-thermal model, and the $\mathrm{BH}$ spin, we found that the best-fit model obtained from the numerical simulations is the one obtained for $R_{\text {low }}=1, R_{\text {high }}=160$, $\sigma_{\text {cut }}=3, \epsilon=0.5$ and $0.5 \lesssim a_{\star} \lesssim 1.0$, respectively. When comparing our results with those already published in the literature to constrain the spin of M87*, we find that there is an overall good agreement, despite the use of different techniques. In particular, some authors find that $a_{\star} \geq 0.4$ is needed to generate a sufficiently large magnetic flux to launch the jet ${ }^{38}$, whereas a higher spin value, i.e., $a_{\star} \sim 0.98$, was deduced when using only the spectral energy distribution of M87* observations, in particular the jet power in the X-ray band, to constrain a jet within an advection-dominated accretion flow (ADAF) model $^{39}$. Finally, our results are also in agreement with the estimates derived by the EHT when accounting for the X-ray luminosity and jet power $\left(\left|a_{\star}\right| \gtrsim 0.5\right)$ (ref. ${ }^{6}$ ), and with the very recent polarised images at $230 \mathrm{GHz}$ recently presented from EHT observations of M87*, where high spins of $0.5 \lesssim a_{\star} \lesssim 1.0$ were favoured $^{40,41}$.

\section{Convolved imaging and comparison with the observations}

To reproduce the GMVA observations, we have convolved the ray-traced images at $86 \mathrm{GHz}$ with an elliptical beam of $307 \times 116 \mu$ as at a position angle of $-9^{\circ}$, which corresponds to the March 2014 GMVA observation of M87 (ref. ${ }^{4}$ ). To obtain the best-fit models presented in the lower row of Fig. 3, we compute the normalised cross-correlation coefficient and the structural dis-similarity image measure (DSSIM) between the GMVA image and the convolved GRRT images ${ }^{27}$.

Furthermore, to compute the jet width, we sliced the jet transversal to the jet axis (defined the direction of propagation) and fitted the flux density profile with two or three different Gaussian distributions. The third Gaussian is typically required to produce a good fit, i.e., $\chi^{2} \sim 1$ (see Extended Data Figure 6). The final jet width is then measured between the peaks of the two outermost Gaussians ${ }^{9}$; the error estimate for the jet width from the GMVA observations, by contrast, is cal- 
culated following the approach of Mertens et al. ${ }^{42}$. For the convolved GRRT images, we computed the mean and standard deviation of the jet width by applying the above described method to our entire data set (see Fig. 4).

Acknowledgements This research is supported by the ERC synergy grant "BlackHoleCam: Imaging the Event Horizon of Black Holes" (Grant No. 610058). CMF is supported by the Black Hole Initiative at Harvard University, which is supported by a grant from the John Templeton Foundation. AN was supported by the Hellenic Foundation for Research and Innovation (H.F.R.I.) under the "2nd Call for H.F.R.I. Research Projects to support Post-Doctoral Researchers" (Project Number: 00634). ZY is supported by a UK Research and Innovation Stephen Hawking Fellowship and acknowledges support from a Leverhulme Trust Early Career Fellowship. JD is supported by NASA grant NNX17AL82 and a Joint Columbia/Flatiron Postdoctoral Fellowship. Research at the Flatiron Institute is supported by the Simons Foundation. The simulations were performed on Goethe-HLR at the Scientific Computing in Frankfurt, on Iboga at the Institut für Theoretische Physik, and Pi2.0 at Shanghai Jiao Tong University.

Author Contributions ACO and CMF performed and analysed the GRRT calculations and wrote the manuscript. YM performed the GRMHD simulations and wrote the manuscript. AN wrote the manuscript. ZY authored the GRRT code BHOS.S. OP authored the GRMHD code BHAC. JD helped in GRRT calculations. H. F. and M. K. wrote the manuscript. LR wrote the manuscript and coordinated the various aspects of the research. All authors discussed the results and commented on all versions of the manuscript.

Correspondence Correspondence and requests for materials should be addressed to Alejandro CruzOsorio (email: osorio@itp.uni-frankfurt.de), Christian M. Fromm (email:cfromm@itp.uni-frankfurt.de) and Yosuke Mizuno (email:mizuno@sjtu.edu.cn).

Data availability The data that support the plots within this paper and other findings of this study are available from the corresponding authors upon reasonable request.

Code availability The public released version of the GRMHD code BHAC can be found at https://bhac.science. The eht-imaging software to convolve the images is available at repository https://github.com/achael/ehtimaging.

Competing Interests The authors declare that they have no competing financial interests. 


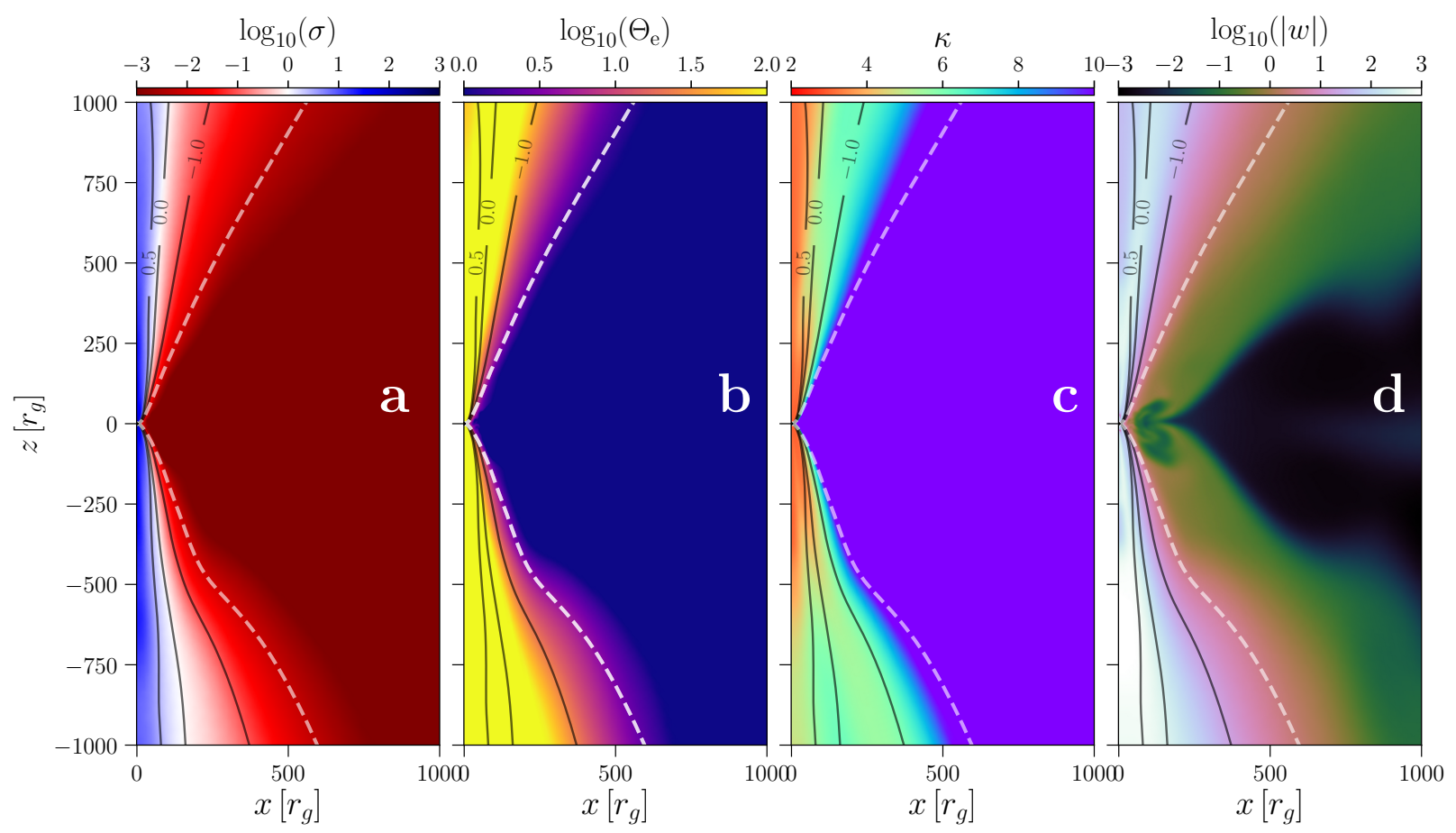

Extended Data Figure 1: Large-scale morphology of the jet from GRMHD simulations of a Kerr BH with spin $\mathrm{a}_{\star}=0.9375$ (the BH spin is aligned with the $z$ axis). a) Shown the magnetisation parameters $\sigma, \mathbf{b})$ the electron temperature $\Theta_{\mathrm{e}}, \mathbf{c}$ ) the distribution of the power-law index $\kappa$, and d) the weighted temperature $w$. All quantities are averaged in space (over the azimuthal direction) and in time (over a time interval of 2,000 $M$ with a cadence of $10 M$ ). The white dashed line marks the boundary between bounded and unbounded $(\mathrm{Be}>1.02)$ material, while the black lines show the most important contours of the logarithm of the magnetisation, $\log _{10} \sigma=-1.0,0.0$, and 0.5. Moving out from the polar axis, we report the contours for the "jet spine", the "jet sheath", and the "external wall". 


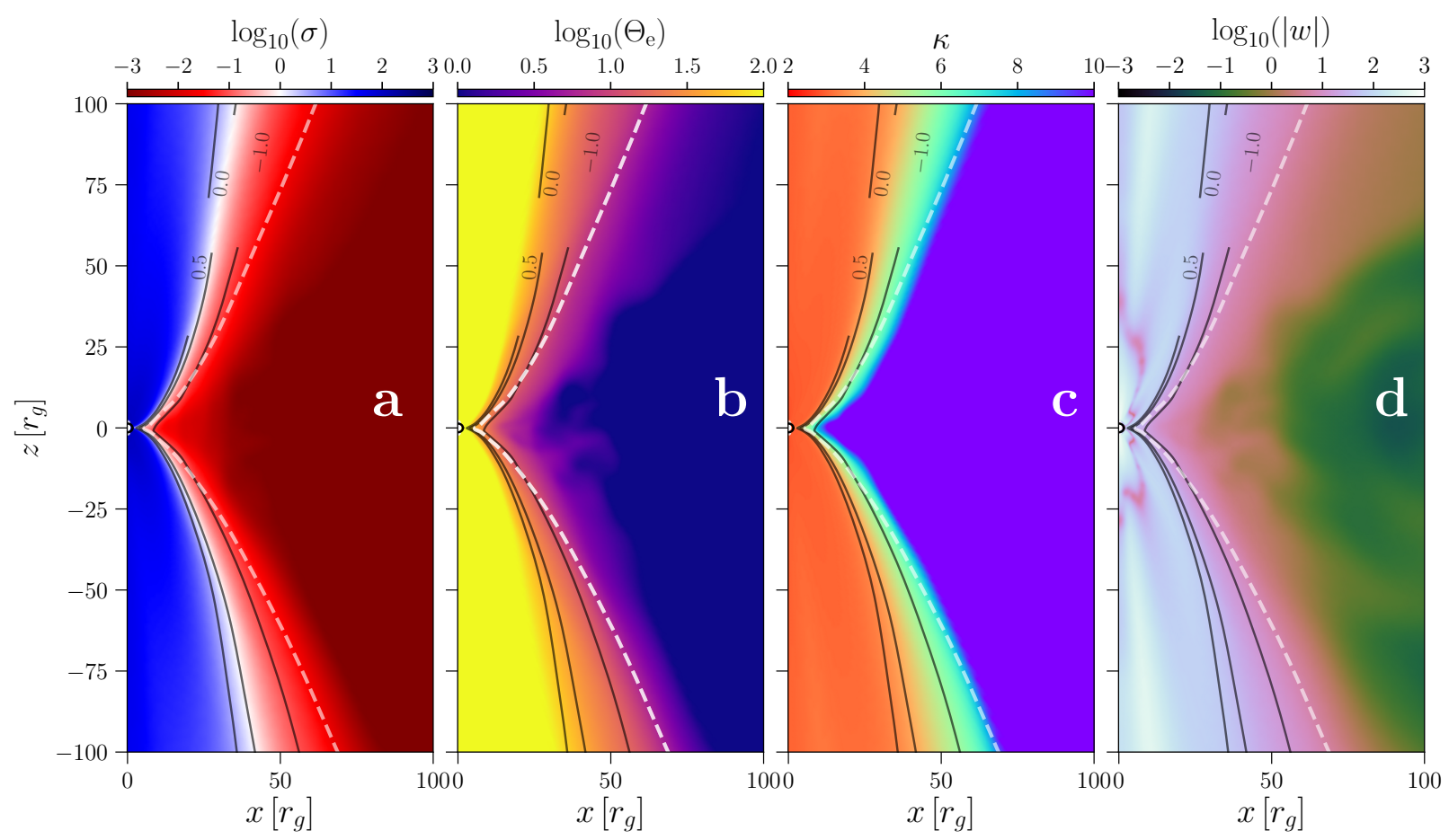

Extended Data Figure 2: Small-scale morphology of the jet from GRMHD simulations. Same as in Extended Data Figure 1, Kerr BH with spin $\mathbf{a}_{\star}=\mathbf{0 . 9 3 7 5}$, but on smaller lengthscales. 


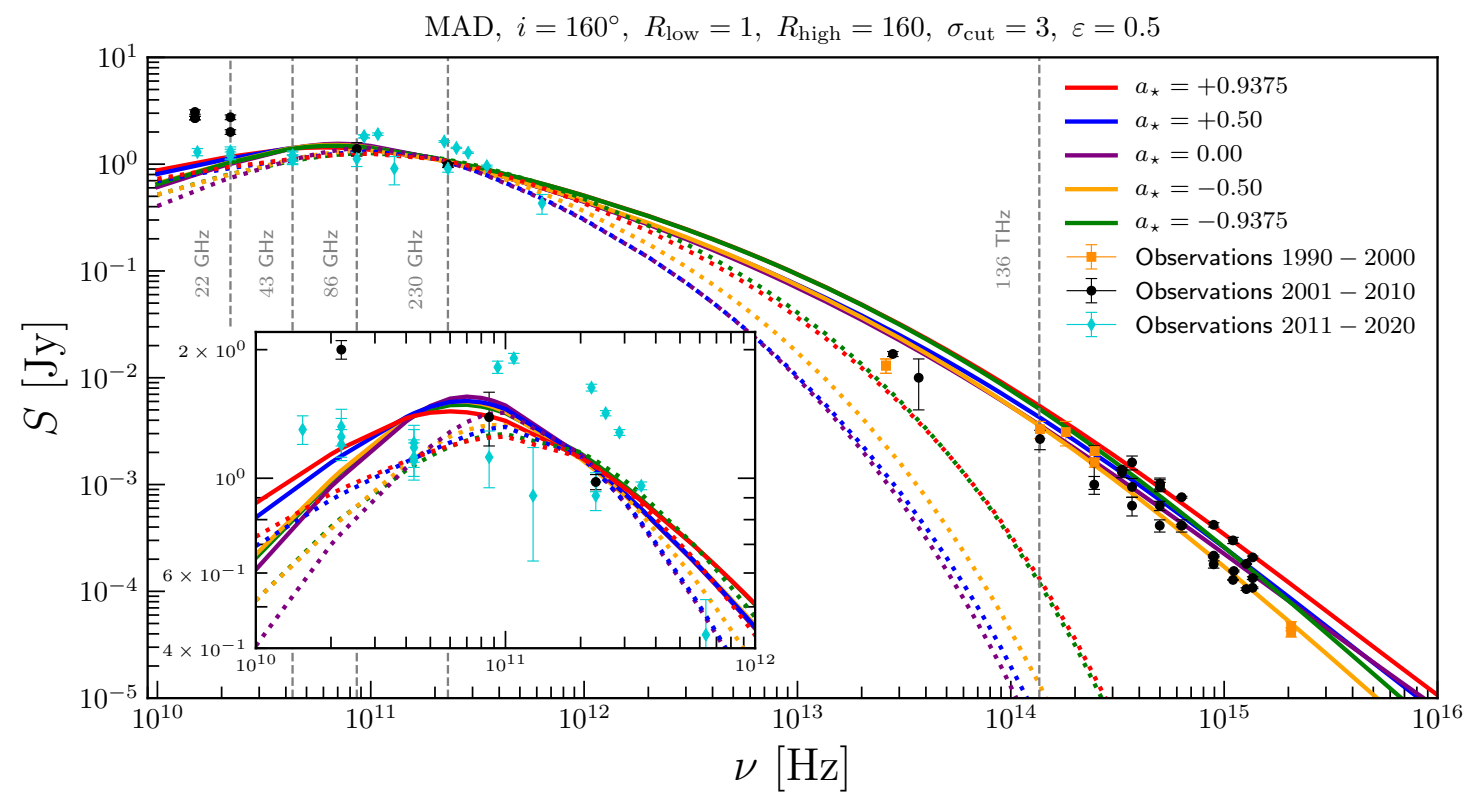

Extended Data Figure 3: Same as in Fig. 2, but considering five different values of the black hole spin. Solid and dotted lines represent nonthermal and thermal emission models, respectively. While gray vertical lines show the most representative frequencies. For each observational data, the uncertainties indicate the variability during the observations. 


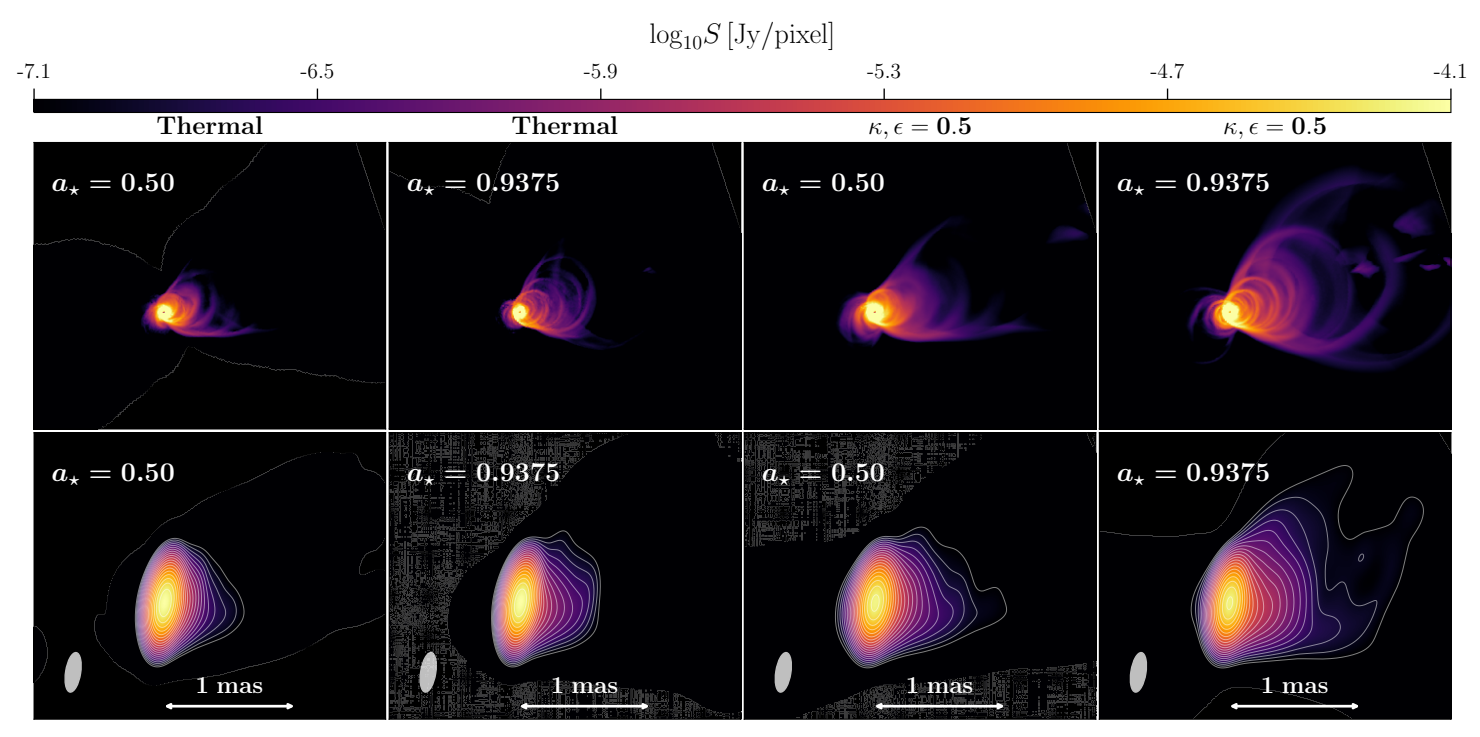

Extended Data Figure 4: GRRT and convolved images. The top panels shown the GRRT, while bottom shown convolved images for thermal and nonthermal emission models for two BHs with spins $\boldsymbol{a}_{\star}=\mathbf{0 . 5 0}$ and $\boldsymbol{a}_{\star}=\mathbf{0 . 9 3 7 5}$. The images refer to a representative time $(t=13,820 \mathrm{M})$ and we show as an ellipse in the lower-left corner the convolving beam with axes $116 \times 307 \mu$ as, as in the observational data. The contours in the flux density correspond to $S_{i}=S_{\min }+0.43 \mathrm{mJy} \times \sqrt{2}^{i}$, where $i=0,1, \ldots, n$, such that $S_{n}<S_{\max }$.

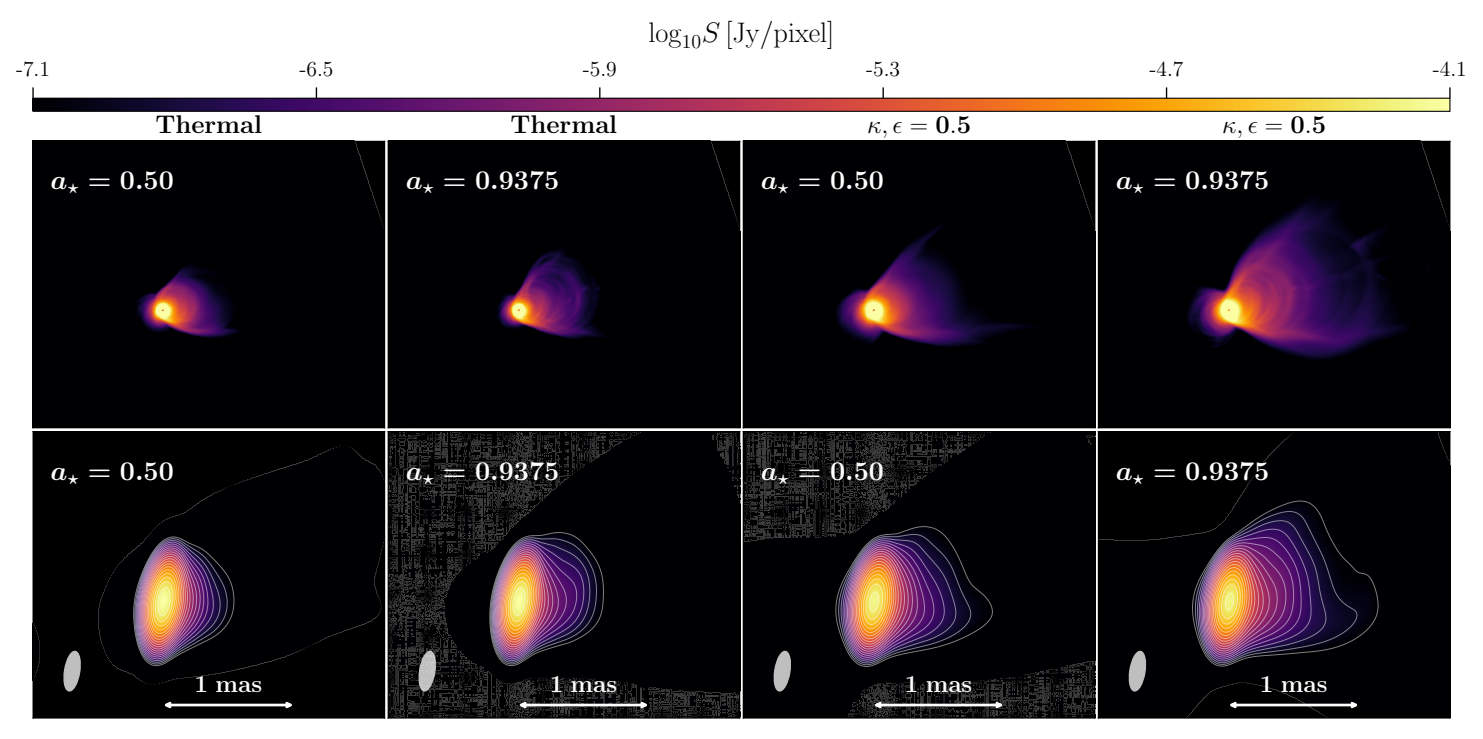

Extended Data Figure 5: Same as in Extended Data Figure 4, but showing the time average computed between $13,000 \mathrm{M}$ and $15,000 \mathrm{M}$. Note that all of the features discussed in Fig. Extended Data Figure 4 are present also when averaging in time. 


\begin{tabular}{r|c}
\hline \multicolumn{1}{c|}{$a_{\star}$} & $\chi^{2}$ \\
\hline-0.9375 & 15.04 \\
-0.5000 & 12.52 \\
0.0000 & 19.53 \\
+0.5000 & 8.51 \\
+0.9375 & 1.46 \\
\hline
\end{tabular}

Extended Data Figure 6: $\chi^{2}$ for the jet width. $\chi^{2}$ between the observational data and the jet width measured from the numerical simulations (see Fig. 4).

1. Kovalev, Y. Y., Lister, M. L., Homan, D. C. \& Kellermann, K. I. The Inner Jet of the Radio Galaxy M87. Astrophys. J. Lett. 668, L27-L30 (2007).

2. Asada, K. \& Nakamura, M. The Structure of the M87 Jet: A Transition from Parabolic to Conical Streamlines. Astrophys. J. l 745, L28 (2012).

3. Walker, R. C., Hardee, P. E., Davies, F. B., Ly, C. \& Junor, W. The Structure and Dynamics of the Subparsec Jet in M87 Based on 50 VLBA Observations over 17 Years at $43 \mathrm{GHz}$. Astrophys. J. 855, 128 (2018).

4. Kim, J. Y. et al. The limb-brightened jet of M87 down to the 7 Schwarzschild radii scale. Astron. Astrophys. 616, A188 (2018).

5. Blandford, R. D. \& Znajek, R. L. Electromagnetic extraction of energy from Kerr black holes. Mon. Not. R. Astron. Soc. 179, 433-456 (1977).

6. Event Horizon Telescope Collaboration et al. First M87 Event Horizon Telescope Results. V. Physical Origin of the Asymmetric Ring. Astrophys. J. Lett. 875, L5 (2019).

7. Narayan, R., Igumenshchev, I. V. \& Abramowicz, M. A. Magnetically Arrested Disk: an Energetically Efficient Accretion Flow. Publications of the ASJ 55, L69-L72 (2003).

8. Narayan, R., Sądowski, A., Penna, R. F. \& Kulkarni, A. K. GRMHD simulations of magnetized advection-dominated accretion on a non-spinning black hole: role of outflows. Mon. Not. R. Astron. Soc. 426, 3241-3259 (2012).

9. Kim, J.-Y. et al. Long-term millimeter VLBI monitoring of M 87 with KVN at milliarcsecond resolution: nuclear spectrum. Astron. Astrophys. 610, L5 (2018).

10. Junor, W. \& Biretta, J. A. The Radio Jet in 3C274 at 0.01 PC Resolution. Astronomical Journal 109, 500 (1995).

11. Ball, D., Sironi, L. \& Özel, F. Electron and Proton Acceleration in Trans-relativistic Magnetic Reconnection: Dependence on Plasma Beta and Magnetization. Astrophys. J. 862, 80 (2018). 
12. Xiao, F. Modelling energetic particles by a relativistic kappa-loss-cone distribution function in plasmas. Plasma Physics and Controlled Fusion 48, 203-213 (2006).

13. Davelaar, J. et al. Modeling non-thermal emission from the jet-launching region of M 87 with adaptive mesh refinement. Astron. Astrophys. 632, A2 (2019).

14. Mizuno, Y. et al. Comparison of the ion-to-electron temperature ratio prescription: GRMHD simulations with electron thermodynamics. Mon. Not. R. Astron. Soc. 506, 741-758 (2021).

15. Younsi, Z., Porth, O., Mizuno, Y., Fromm, C. M. \& Olivares, H. Modelling the polarised emission from black holes on event horizon-scales. In Asada, K., de Gouveia Dal Pino, E., Giroletti, M., Nagai, H. \& Nemmen, R. (eds.) Perseus in Sicily: From Black Hole to Cluster Outskirts, vol. 342, 9-12 (2020).

16. Mościbrodzka, M., Falcke, H., Shiokawa, H. \& Gammie, C. F. Observational appearance of inefficient accretion flows and jets in 3D GRMHD simulations: Application to Sa gittarius A*. Astron. Astrophys. 570, A7 (2014).

17. Doeleman, S. S. et al. Jet-Launching Structure Resolved Near the Supermassive Black Hole in M87. Science 338, 355 (2012).

18. Perlman, E. S. et al. Deep 10 Micron Imaging of M87. Astrophys. J. Lett. 561, L51-L54 (2001).

19. Lonsdale, C. J., Doeleman, S. S. \& Phillips, R. B. A 3 Millimeter VLBI Continuum Source Survey. Astronomical Journal 116, 8-12 (1998).

20. Whysong, D. \& Antonucci, R. Thermal Emission as a Test for Hidden Nuclei in Nearby Radio Galaxies. Astrophys. J. 602, 116-122 (2004).

21. Akiyama, K. et al. $230 \mathrm{GHz}$ VLBI Observations of M87: Event-horizon-scale Structure during an Enhanced Very-high-energy $\gamma$-Ray State in 2012. Astrophys. J. 807, 150 (2015).

22. Prieto, M. A., Fernández-Ontiveros, J. A., Markoff, S., Espada, D. \& González-Martín, O. The central parsecs of M87: jet emission and an elusive accretion disc. Mon. Not. R. Astron. Soc. 457, 3801-3816 (2016).

23. Hada, K. et al. Pilot KaVA monitoring on the M 87 jet: Confirming the inner jet structure and superluminal motions at sub-pc scales. Publications of the ASJ 69, 71 (2017).

24. An, T., Sohn, B. W. \& Imai, H. Capabilities and prospects of the East Asia Very Long Baseline Interferometry Network. Nature Astronomy 2, 118-125 (2018).

25. Lister, M. L. et al. MOJAVE. XV. VLBA $15 \mathrm{GHz}$ Total Intensity and Polarization Maps of 437 Parsec-scale AGN Jets from 1996 to 2017. Astrophys. J., Supp. 234, 12 (2018).

26. Nakamura, M. et al. Parabolic Jets from the Spinning Black Hole in M87. Astrophys. J. 868, 146 (2018). 
27. Mizuno, Y. et al. The current ability to test theories of gravity with black hole shadows. Nature Astronomy 2, 585-590 (2018).

28. Porth, O. et al. The black hole accretion code. Computational Astrophysics and Cosmology 4, 1 (2017).

29. Rezzolla, L. \& Zanotti, O. Relativistic Hydrodynamics (Oxford University Press, Oxford, UK, 2013).

30. Olivares, H. et al. Constrained transport and adaptive mesh refinement in the Black Hole Accretion Code. Astron. Astrophys. 629, A61 (2019).

31. Font, J. A. \& Daigne, F. On the stability of thick accretion disks around black holes. Astrophys. J 581, L23-L26 (2002).

32. Shiokawa, H., Dolence, J. C., Gammie, C. F. \& Noble, S. C. Global General Relativistic Magnetohydrodynamic Simulations of Black Hole Accretion Flows: A Convergence Study. Astrophys. J. 744, 187 (2012).

33. Gold, R., Broderick, A. E., Younsi, Z., Fromm, C. M. \& et al. Verification of Radiative Transfer Schemes for the EHT. Astrophys. J. 897, 148 (2020).

34. Younsi, Z., Wu, K. \& Fuerst, S. V. General relativistic radiative transfer: formulation and emission from structured tori around black holes. Astron. Astrophys. 545, A13 (2012).

35. Event Horizon Telescope Collaboration et al. First M87 Event Horizon Telescope Results. I. The Shadow of the Supermassive Black Hole. Astrophys. J. Lett. 875, L1 (2019).

36. Mościbrodzka, M., Gammie, C. F., Dolence, J. C., Shiokawa, H. \& Leung, P. K. Radiative Models of SGR A* from GRMHD Simulations. Astrophys. J. 706, 497-507 (2009).

37. Chael, A., Narayan, R. \& Johnson, M. D. Two-temperature, Magnetically Arrested Disc simulations of the jet from the supermassive black hole in M87. Mon. Not. R. Astron. Soc. 486, 2873-2895 (2019).

38. Nemmen, R. The Spin of M87*. Astrophys. J. Lett. 880, L26 (2019).

39. Feng, J. \& Wu, Q. Constraint on the black hole spin of M87 from the accretion-jet model. Mon. Not. R. Astron. Soc. 470, 612-616 (2017).

40. Event Horizon Telescope Collaboration et al. First M87 Event Horizon Telescope Results. VII. Polarization of the Ring. Astrophys. J. Lett. 910, L12 (2021).

41. Event Horizon Telescope Collaboration et al. First M87 Event Horizon Telescope Results. VIII. Magnetic Field Structure near The Event Horizon. Astrophys. J. Lett. 910, L13 (2021).

42. Mertens, F., Lobanov, A. P., Walker, R. C. \& Hardee, P. E. Kinematics of the jet in M 87 on scales of 100-1000 Schwarzschild radii. Astron. Astrophys. 595, A54 (2016). 